\title{
Laparoscopic image of carbon dioxide embolism during laparoscopic hepatectomy: a case report
}

\author{
Kenichi Takechi* ${ }^{*}$, Mari Ito, Yi Peng, Waka Daizen and Ichiro Shimizu
}

\begin{abstract}
Background: Carbon dioxide embolism is a life-threatening complication of laparoscopic hepatectomy.

Case presentation: A 59-year-old man was admitted for laparoscopic hepatectomy. Approximately $5 \mathrm{~h}$ after commencing the operation, we observed a gradual decline in the $\mathrm{SpO}_{2}$ from 100 to 94\%, reduction in the $\mathrm{ETCO}_{2}$ from 44 to $19 \mathrm{mmHg}$, reduction in the systolic blood pressure from 100 to $82 \mathrm{mmHg}$, and elevation of the heart rate from 82 to 120 beats/min. Intraoperatively, the image displayed on the laparoscopic monitor revealed a small tear in the vein. The inspired $\mathrm{O}_{2}$ fraction was raised to 1.0 , intravenous phenylephrine ( $0.1 \mathrm{mg}$ bolus) was administered, and the respiratory rate was increased. After the patient was stabilized, the injured vein was cut and sealed. After the embolic event, the entire operation was completed without complications.

Conclusions: Careful observation of the laparoscopic monitor is important, particularly during establishment of pneumoperitoneum in patients undergoing laparoscopic hepatectomy.
\end{abstract}

Keywords: Pulmonary embolism, Laparoscopy complications, Pringle maneuver

\section{Background}

Compared with open hepatectomy, laparoscopic hepatectomy is a minimally invasive procedure associated with lesser intraoperative bleeding and postoperative pain and faster recovery. Carbon dioxide $\left(\mathrm{CO}_{2}\right)$ is commonly used for pneumoperitoneum during laparoscopic procedures, and $\mathrm{CO}_{2}$ embolism is an uncommon but life-threatening complication of laparoscopic hepatectomy [1]. Low central venous pressure $(<5 \mathrm{mmHg})$, high-pressure pneumoperitoneum $(>12 \mathrm{mmHg})$, and the Pringle maneuver are used to achieve hemostasis during laparoscopic hepatectomy [2]. However, intra-abdominal insufflation pressures exceeding the central venous pressure theoretically predispose patients to $\mathrm{CO}_{2}$ embolism, particularly when performing operations involving highly vascular solid organs such as the liver. Reportedly, the

\footnotetext{
* Correspondence: ntakechi@me.com

Support was provided solely from institutional and/or departmental

sources.The authors declare no competing interests.

Matsuyama Red Cross Hospital, 1 Bunkyocho, Matsuyama City, Ehime, Japan
}

incidence of $\mathrm{CO}_{2}$ embolism during laparoscopic hepatectomy (1.2-4.6\%) was approximately 10-fold higher than the overall incidence of $\mathrm{CO}_{2}$ embolism during laparoscopic surgery $(0.15 \%)[1,3,4]$. Usually, dissociation between the end-tidal carbon dioxide $\left(\mathrm{ETCO}_{2}\right)$ and arterial gas $\mathrm{CO}_{2}$ is used to diagnose $\mathrm{CO}_{2}$ embolism. We describe $\mathrm{CO}_{2}$ embolism that gas entry was confirmed on a laparoscopic monitor in a patient who underwent laparoscopic hepatectomy using the Pringle maneuver.

\section{Case presentation}

A 59-year-old man (height $171 \mathrm{~cm}$, weight $74 \mathrm{~kg}$ ) was admitted to our hospital for laparoscopic right hemihepatectomy for a diagnosis of intrahepatic cholangiocarcinoma. The operation was performed under combined general and epidural anesthesia. Preoperative laboratory findings were within normal limits, and no premedication was administered to the patient. A standard anesthetic protocol was implemented, including routine non-invasive arterial blood pressure monitoring,

\section{Springer Open}

(อ) The Author(s). 2020 Open Access This article is licensed under a Creative Commons Attribution 4.0 International License, which permits use, sharing, adaptation, distribution and reproduction in any medium or format, as long as you give appropriate credit to the original author(s) and the source, provide a link to the Creative Commons licence, and indicate if changes were made. The images or other third party material in this article are included in the article's Creative Commons licence, unless indicated otherwise in a credit line to the material. If material is not included in the article's Creative Commons licence and your intended use is not permitted by statutory regulation or exceeds the permitted use, you will need to obtain permission directly from the copyright holder. To view a copy of this licence, visit http://creativecommons.org/licenses/by/4.0/. 
electrocardiography, and oxygen saturation $\left(\mathrm{SpO}_{2}\right)$ measurement on arrival at the operating room, and his vital signs were stable. Before induction of general anesthesia, a thoracic epidural catheter was inserted into the thoracic 7-8 interspace. Anesthesia was induced using propofol $(2 \mathrm{mg} / \mathrm{kg})$, remifentanil $(0.3 \mu \mathrm{g} / \mathrm{kg} / \mathrm{min})$, and rocuronium $(0.8 \mathrm{mg} / \mathrm{kg})$ and was maintained using desflurane (4-6\%), remifentanil $(0.15-0.3 \mu \mathrm{g} / \mathrm{kg} / \mathrm{min})$, and rocuronium $(5 \mu \mathrm{g} / \mathrm{kg} / \mathrm{min})$. The following ventilator settings were used: tidal volume $7 \mathrm{~mL} / \mathrm{kg}$ ideal body weight, inspiratory:expiratory ratio $1: 2$, inspired $\mathrm{O}_{2}$ fraction 0.6 with air, and inspiratory fresh gas flow $2 \mathrm{~L} / \mathrm{min}$. The respiratory rate was adjusted to $8-16$ breaths/min to maintain an $\mathrm{ETCO}_{2}$ pressure of $30-45 \mathrm{mmHg}$. After induction of anesthesia and tracheal intubation, a 22-G catheter was inserted into the left radial artery for blood sampling and continuous blood pressure monitoring. A central venous catheter was inserted from the right jugular vein for continuous central venous pressure monitoring. The patient was placed in the reverse Trendelenburg position with a left tilt, and $\mathrm{CO}_{2}$ was insufflated to create pneumoperitoneum (intra-abdominal pressure $12 \mathrm{mmHg}$ ). A low central venous pressure $(<5 \mathrm{mmHg}$ ) was maintained throughout the operation.

Approximately $5 \mathrm{~h}$ after commencing the operation and establishment of pneumoperitoneum, during the Pringle maneuver (hepatic resection performed with clamping the branches of the vascular pedicle), we observed a gradual decline in the patient's $\mathrm{SpO}_{2}$ from 100 to $94 \%$, reduction in the $\mathrm{ETCO}_{2}$ from 44 to 19 $\mathrm{mmHg}$, reduction in the systolic blood pressure from 100 to $82 \mathrm{mmHg}$, and rapid elevation of the heart rate from 82 to 120 beats/min (Fig. 1).
Intraoperatively, the image displayed on the laparoscopic monitor revealed a small tear in the wall of a vein without bleeding but ballooning with gas within the vessel (Fig. 2, Supplementary video 1). $\mathrm{CO}_{2}$ embolism was suspected, and arterial blood gas testing was performed for confirmation ( $\mathrm{pH} 7.153$, partial pressure of $\mathrm{O}_{2} 122.8 \mathrm{mmHg}$, partial pressure of $\mathrm{CO}_{2}$ $78.2 \mathrm{mmHg}$ ). The pneumoperitoneum pressure was reduced to $10 \mathrm{mmHg}$, and the Pringle maneuver was abandoned. The inspired $\mathrm{O}_{2}$ fraction was immediately raised to 1.0 , and intravenous phenylephrine $(0.1 \mathrm{mg}$ bolus) was administered to treat hypotension. The respiratory rate was increased to wash out $\mathrm{CO}_{2}$. After the patient was hemodynamically stabilized, the injured vein was immediately cut and sealed. Approximately $4 \mathrm{~h}$ after this embolic event, the entire operation was completed without complications. Intraoperative blood loss was $550 \mathrm{ml}$, and the patient was safely extubated. His postoperative course was uneventful, and he was discharged without further complications.

\section{Discussion}

In this case, $\mathrm{CO}_{2}$ embolism occurred during establishment of pneumoperitoneum in a patient undergoing the Pringle maneuver. Optimal hemostasis is extremely important during laparoscopic hepatectomy [5]; notably, specific hemostatic modalities are being developed for laparoscopic hepatectomy. High-pressure pneumoperitoneum $(10-14 \mathrm{mmHg})$, low central venous pressure $(<5$ $\mathrm{mmHg}$ ), a carefully controlled pneumoperitoneum gas delivery system, and the use of appropriate hemostatic devices are recommended for safe laparoscopic

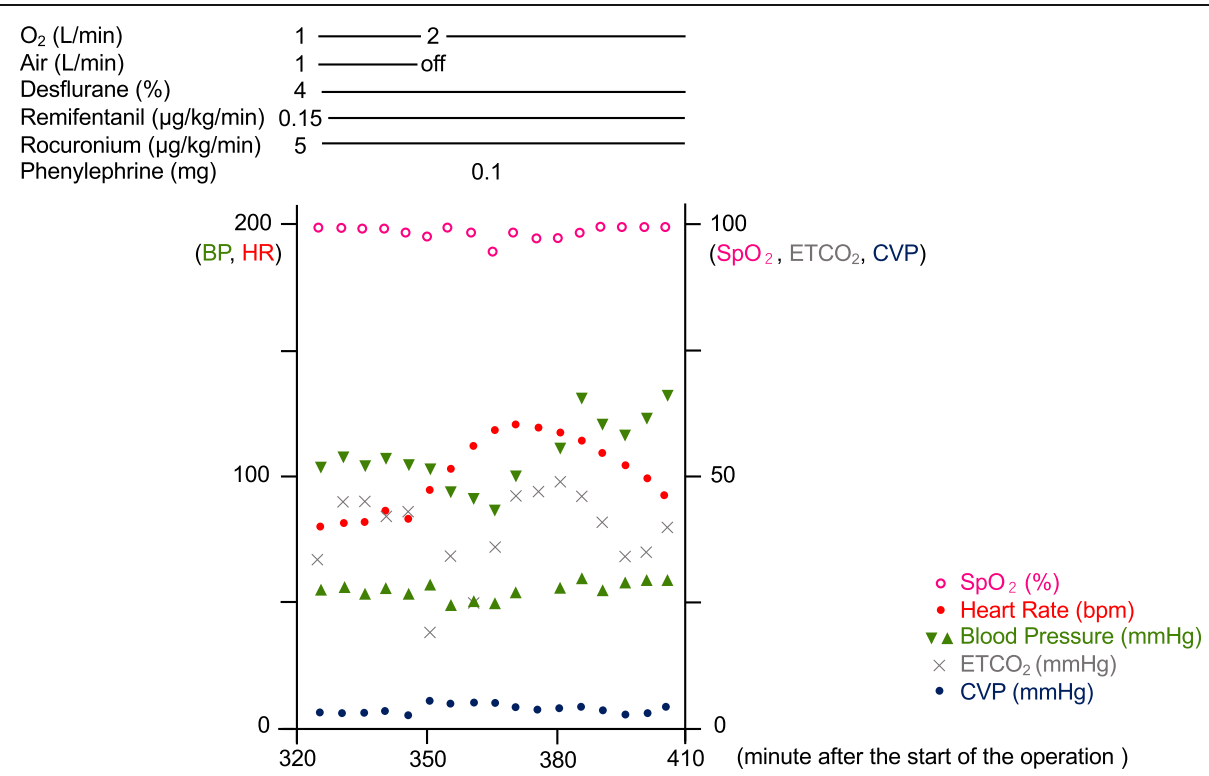

Fig. 1 Vital signs during the $\mathrm{CO}_{2}$ embolism 


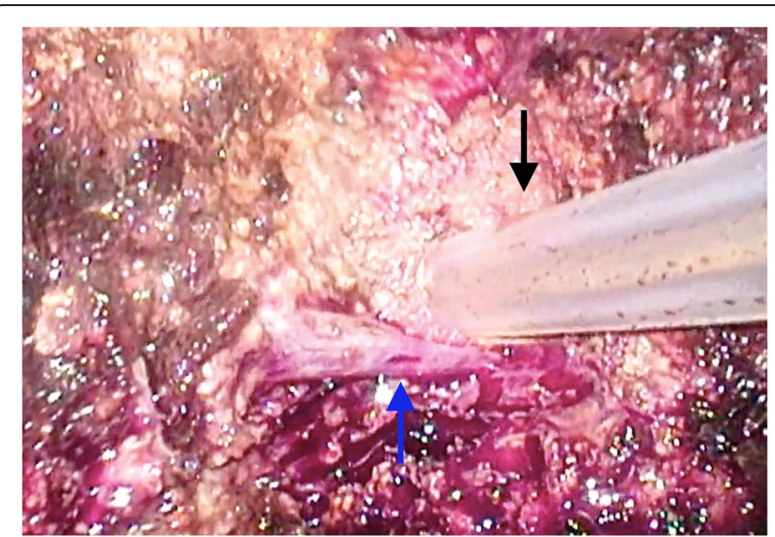

Fig. 2 Laparoscopic image of the surgical field. A small laceration without bleeding is detectable in the wall of a vein on the surface of the resected right hepatic lobe (blue arrow) during surgery using an ultrasonic surgical aspirator (black arrow)

hepatectomy with minimal bleeding [2]. However, low central venous pressure with high-pressure pneumoperitoneum results in a pressure gradient that predisposes patients to $\mathrm{CO}_{2}$ embolism. Clamping the hepatoduodenal ligament interrupts blood flow through the hepatic artery and the portal vein and controls bleeding from the liver. This hemostatic technique, referred to as the Pringle maneuver is commonly used during laparoscopic hepatectomy. During the Pringle maneuver, bleeding occurs secondary to backflow from the hepatic veins, and intrahepatic vascular pressure is usually the same as the central venous pressure. Makabe et al. reported that hepatic artery occlusion increases the risk of $\mathrm{CO}_{2} \mathrm{em}$ bolism during laparoscopic hepatectomy in a pig model [6]. The low central venous pressure, high-pressure pneumoperitoneum, and the Pringle maneuver all contributed to $\mathrm{CO}_{2}$ embolism in our patient. Clinicians should be mindful of the risk of $\mathrm{CO}_{2}$ embolism, particularly during establishment of pneumoperitoneum in patients undergoing surgical procedures that involve the Pringle maneuver.

$\mathrm{CO}_{2}$ embolism typically manifests with a sudden decrease in $\mathrm{ETCO}_{2}$, reduction in blood pressure, and elevated heart rate. Transesophageal echocardiography (TEE) is one of the most sensitive and specific diagnostic modalities for intraoperative $\mathrm{CO}_{2}$ embolism [7]. However, TEE is not routinely used for laparoscopic hepatectomy owing to its invasiveness. Therefore, intraoperative $\mathrm{CO}_{2}$ embolism is usually diagnosed based on clinical symptoms. Using a high-resolution laparoscopic camera, even minute structures in the operative field could be projected as a wide and clear image on a high-resolution monitor in our case. Therefore, the surgeons and anesthesiologist could immediately detect the small tear in the wall of the injured vein. These images facilitated prompt diagnosis of $\mathrm{CO}_{2}$ embolism by the surgical team.
Patients with suspected $\mathrm{CO}_{2}$ embolism should be ventilated using inspired $\mathrm{O}_{2}$ fraction 1.0 to absorb the bubbles and combat the ventilation mismatch to improve hypoxemia [8]. The pneumoperitoneum pressure should be reduced or conversion to open surgery is warranted to release intra-abdominal gas and prevent further embolism [8]. In this case, the surgical team concurred that abandoning the pneumoperitoneum could increase the risk of bleeding from the vein with consequent difficulty in treating the injured vein. Releasing the Pringle maneuver could theoretically increase hepatic blood flow and reduce the pneumoperitoneum pressure-venous pressure gradient. Therefore, we reduced the pneumoperitoneum pressure, abandoned the Pringle maneuver, and continued the laparoscopic procedure. Another possible strategy to prevent progressive $\mathrm{CO}_{2}$ embolism is patient positioning (the Trendelenburg position with a left tilt) and high positive end-expiratory pressure (PEEP). ventilation [9]. However, the Trendelenburg position may interfere with optimal visualization of the operative field during laparoscopic hepatectomy, and high PEEP ventilation may increase the right atrial pressure with a risk of paradoxical embolism through a latent patent foramen ovale [10].

\section{Conclusion}

The Pringle maneuver performed during laparoscopic hepatectomy may theoretically predispose patients to $\mathrm{CO}_{2}$ embolism. Laparoscopic imaging provides important information to enable prompt diagnosis and treatment of $\mathrm{CO}_{2}$ embolism by facilitating effective communication between the surgeon and anesthesiologist. Careful observation of the laparoscopic monitor and rapid communication between the surgeon and the anesthesiologist are important, particularly during the establishment of pneumoperitoneum in patients undergoing operations that involve the Pringle maneuver.

\section{Supplementary information}

Supplementary information accompanies this paper at https://doi.org/10. 1186/s40981-020-00345-4.

Additional file 1:. Supplementary video

\section{Abbreviations}

$\mathrm{CO}_{2}$ : Carbon dioxide; $\mathrm{ETCO}_{2}$ : End-tidal carbon dioxide; $\mathrm{SpO}_{2}$ : Oxygen saturation; PEEP: Positive end-expiratory pressure; TEE: Transesophageal echocardiography

\section{Acknowledgements}

None

Authors' contributions

$\mathrm{KT}$ and $\mathrm{MI}$ were involved in patient management. $\mathrm{KT}$ wrote the manuscript YP, WD, and IS revised the manuscript. All authors read and approved the final manuscript. 


\section{Funding}

Nothing to declare

\section{Availability of data and materials \\ Not applicable}

Ethics approval and consent to participate

Not applicable

\section{Consent for publication}

Written informed consent was obtained from the patient for publication of this case report.

\section{Competing interests}

No external funding and no competing interests declared

Received: 30 April 2020 Accepted: 18 May 2020

Published online: 30 May 2020

\section{References}

1. Otsuka Y, Katagiri T, Ishii J, Maeda T, Kubota Y, Tamura A, et al. Gas embolism in laparoscopic hepatectomy: what is the optimal pneumoperitoneal pressure for laparoscopic major hepatectomy? J Hepatobiliary Pancreat Sci. 2013;20:137-40.

2. Tranchart H, O'Rourke N, Van Dam R, Gaillard M, Lainas P, et al. Bleeding control during laparoscopic liver resection: a review of literature. J Hepatobiliary Pancreat Sci. 2015;22:371-8.

3. Bryant $R$, Laurent $A$, Tayar $C$, Cherqui D. Laparoscopic liver resectionunderstanding its role in current practice: the Henri Mondor Hospital experience. Ann Surg. 2009;250:103-11.

4. Dagher I, Di Giuro G, Dubrez J, Lainas P, Smadja C, Franco D. Laparoscopic versus open right hepatectomy: a comparative study. Am J Surg. 2009;198: $173-7$.

5. Abu Hilal M, Underwood T, Taylor MG, Hamdan K, Elberm H, Pearce NW. Bleeding and hemostasis in laparoscopic liver surgery. Surg Endosc. 2010;24: $572-7$.

6. Makabe K, Nitta H, Takahara T, Hasegawa Y, Kanno S, Nishizuka S, et al. Efficacy of occlusion of hepatic artery and risk of carbon dioxide gas embolism during laparoscopic hepatectomy in a pig model. J Hepatobiliary Pancreat Sci. 2014;21:592-8

7. Couture P, Boudreault D, Derouin M, Allard M, Lepage Y, Girard D, et al. Venous carbon dioxide embolism in pigs: an evaluation of end-tidal carbon dioxide, transesophageal echocardiography, pulmonary artery pressure, and precordial auscultation as monitoring modalities. Anesth Analg. 1994;79: 867-73.

8. Webber S, Andrzejowski J, Francis G. Gas embolism in anaesthesia. BJA CEPD Reviews. 2002;2:53-7.

9. Mirski MA, Lele AV, Fitzsimmons L, Toung TJ. Diagnosis and treatment of vascular air embolism. Anesthesiology. 2007;106:164-77.

10. Kobayashi A, Shirozu K, Karashima Y, Matsushita K, Yamaura K. Cerebra infarction detected after laparoscopic partial hepatectomy: case report. JA Clin Rep. 2019;12:82

\section{Publisher's Note}

Springer Nature remains neutral with regard to jurisdictional claims in published maps and institutional affiliations.

\section{Submit your manuscript to a SpringerOpen ${ }^{\circ}$ journal and benefit from:}

- Convenient online submission

- Rigorous peer review

- Open access: articles freely available online

- High visibility within the field

- Retaining the copyright to your article

Submit your next manuscript at $\boldsymbol{\nabla}$ springeropen.com 